\title{
Tau physics results and prospects at Belle II
}

\section{A. De Yta-Hernández ${ }^{a, *}$ on behalf of the Belle II Collaboration}

${ }^{a}$ Centro de Investigacion y de Estudios Avanzados del Instituto Politecnico Nacional, Av. IPN 2508, San Pedro Zacatenco, Mexico City, Mexico, 07360.

E-mail: adeyta@fis.cinvestav.mx

The Belle II experiment operates at the SuperKEKB energy-asymmetric $e^{+} e^{-}$-collider. Belle II is a substantial upgrade of the Belle detector and has a broad $\tau$ physics program, in particular in searches for lepton flavor and lepton number violations (LFV and LNV). We expect that after 5 years of data taking, Belle II will be able to improve the sensitivity on LFV and LNV $\tau$ decays by an order of magnitude. In this talk we review the $\tau$ physics program of Belle II.

*** The European Physical Society Conference on High Energy Physics (EPS-HEP2021), ***

*** 26-30 July $2021 * * *$

*** Online conference, jointly organized by Universität Hamburg and the research center DESY ***

${ }^{*}$ Speaker 


\section{Introduction}

The $\tau$ lepton is the heaviest lepton in the Standard Model (SM) of particle physics. In fact, it is the only lepton heavy enough to decay into leptons or hadrons, as shown in Figure 1. Since the mechanisms that govern its production and decay in electroweak interactions are well-understood, the physics of the $\tau$ lepton constitutes an ideal tool to rigorously test the SM and search for physics beyond the Standard Model (BSM). $B$ factories offer rich possibilities to study $\tau$ physics. In particular, the Belle II experiment expects to collect about 45 billion of $\tau^{+} \tau^{-}$pairs in its full data set, enabling a broad $\tau$ physics program. Because of the unique detection capabilities of the Belle II detector and the unprecedented amount of data to be collected by the experiment in the coming years, it will be possible to perform precise measurements of the $\tau$ properties and accurate determinations of its parameters. In addition, we expect that Belle II will provide important results on searches for SM suppressed lepton flavor violating (LFV) and lepton number violating (LNV) processes in the $\tau$ sector, whose observation would be the evidence of BSM scenarios, offering the opportunity to probe the underlying dynamics.

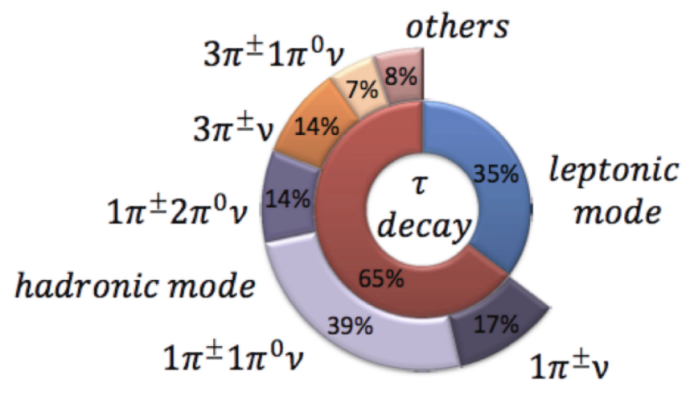

Figure 1: $\tau$ lepton decay modes.

We report the current status of two important analyses, the measurement of the $\tau$ lepton mass, and the search for the LFV decay channel $\tau \rightarrow \ell \alpha$, where $\ell$ is either an electron or a muon, and $\alpha$ is a massive BSM particle that escapes undetected. Additionally, we comment on the prospects of searches for LFV decays of the $\tau$ lepton.

\section{SuperKEKB Collider and the Belle II Experiment}

SuperKEKB is a $7 \mathrm{GeV}$ electron $-4 \mathrm{GeV}$ positron collider at the KEK laboratory in Tsukuba, Japan [1]. Figure 2 shows a schematic representation of the SuperKEKB facility. The target instantaneous luminosity of SuperKEKB is $6 \times 10^{35} \mathrm{~cm}^{2} \mathrm{~s}^{-1}$, a factor of 30 greater than KEKB, and the goal is to accumulate an integrated luminosity of $50 \mathrm{ab}^{-1}$ in the next decade. To date, Belle II has reached an instantaneous luminosity of $3.1 \times 10^{34} \mathrm{~cm}^{-2} \mathrm{~s}^{-1}$ and collected an integrated luminosity of $213 \mathrm{fb}^{-1}$.

Belle II is an upgraded version of its predecessor experiment, Belle, and includes some completely new subdetectors, as well as improvements to others. The Belle II detector [2] has a cylindrical structure, and consists of several subdetectors as shown in Figure 2. 

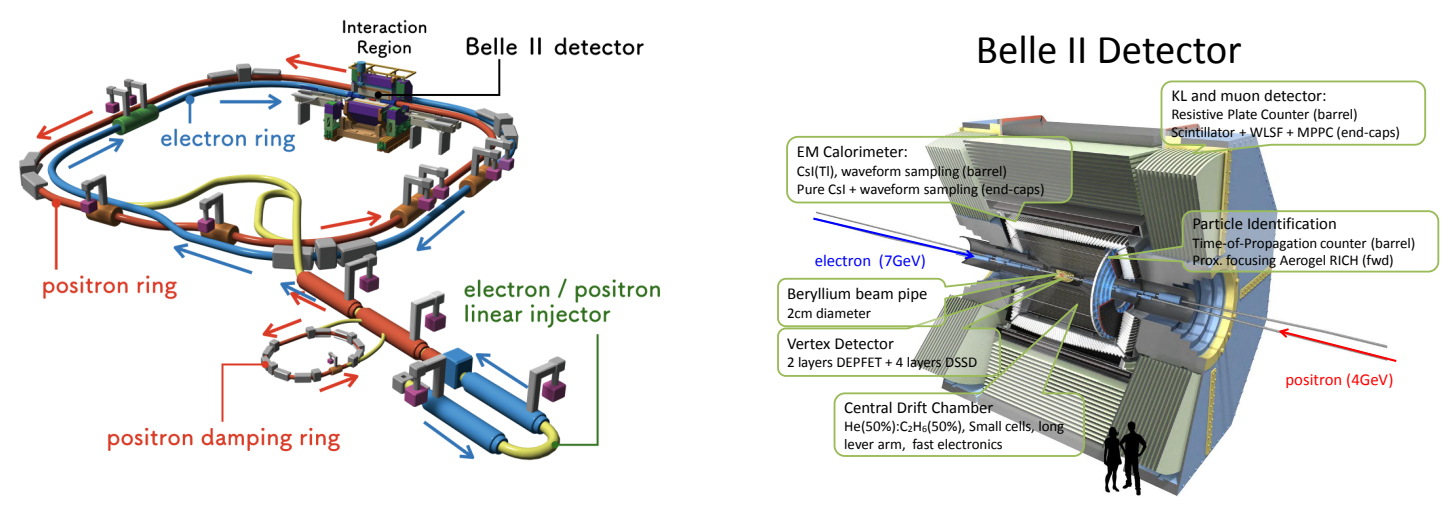

Figure 2: Schematic drawing of SuperKEKB facility and Belle II subdetector composition.

\section{Tau Mass Measurement}

In the SM, the masses of quarks and leptons are fundamental parameters, which cannot be determined by theory and must be measured. Precision measurements of the $\tau$ lepton mass are important for testing lepton universality and for calculating branching fractions that depend on the $\tau$ mass [3].

A first measurement of the $\tau$ lepton mass at Belle II is performed following the so-called pseudomass method introduced by the ARGUS collaboration [4]. We use 3x1 prong events, where the signal is the decay $\tau \rightarrow 3 \pi v$, and the other pair-produced $\tau$ decays into a 1 prong system. For this configuration, the pseudomass is given by

$$
M_{\mathrm{min}}=\sqrt{M_{3 \pi}^{2}+2\left(E_{\mathrm{beam}}-E_{3 \pi}\right)\left(E_{3 \pi}-P_{3 \pi}\right)}
$$

where $M_{3 \pi}, P_{3 \pi}, E_{3 \pi}$ are the invariant mass, the magnitude of the three-momentum and the energy of the $3 \pi$ system; and $E_{\text {beam }}$ is the beam energy in the $e^{+} e^{-}$center-of-mass frame (CM), respectively.

In order to estimate the $\tau$ lepton mass $\left(m_{\tau}\right)$, the following empirical probability density function (p.d.f.) is fitted to the data in the pseudomass region from 1.7 to $1.85 \mathrm{GeV} / c^{2}$ :

$$
F\left(M_{\min }, \vec{P}\right)=\left(P_{3}+P_{4} M_{\min }\right) \arctan \left[\left(M_{\min }-P_{1}\right) / P_{2}\right]+P_{5} M_{\min }+1
$$

where $P_{1}$ is the estimator of $m_{\tau}$ and the parameters $P_{2}-P_{5}$ are free parameters. The fit determines $m_{\tau}=1777.28 \pm 0.75 \mathrm{MeV} / c^{2}$. Figure 3 shows the pseudomass distribution for $\tau \rightarrow 3 \pi v$ candidates, and the fit results [5]. A kinematic cutoff appears at $M_{\min } \approx m_{\tau}$.

The Belle II result agrees with previous measurements [5]. Belle II has similar systematic error as Belle, as shown in Figure 3. A scenario with a reduced total systematic uncertainty is expected in the near future. 

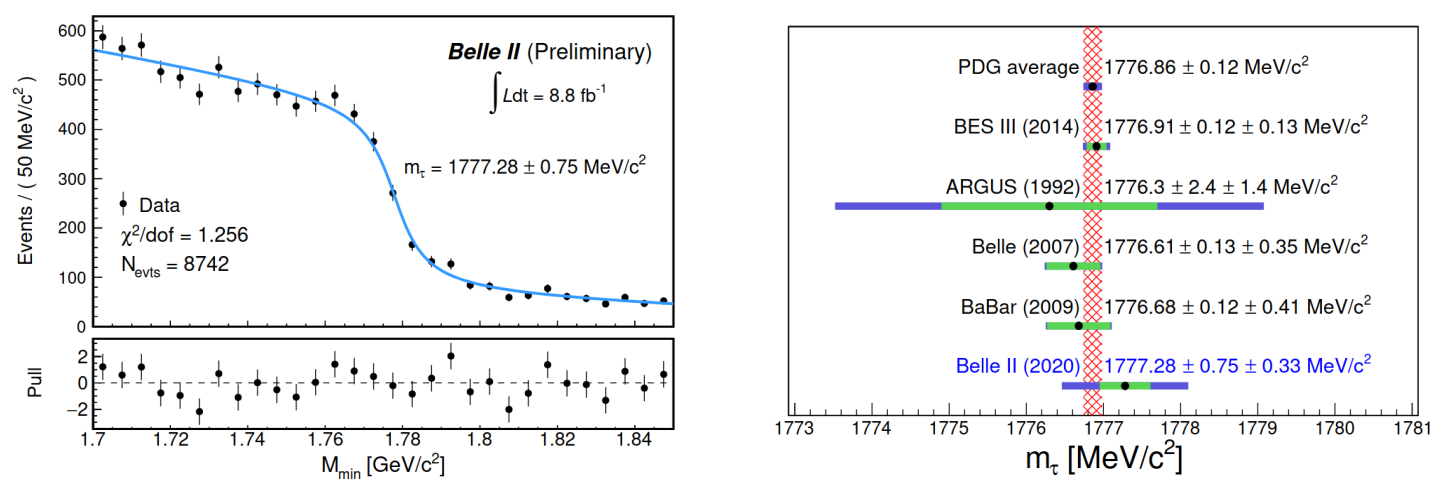

Figure 3: Distribution of pseudomass in a sample of Belle II data (points with error bars) corresponding to an integrated luminosity of $8.8 \mathrm{fb}^{-1}$ with fit projections overlaid (solid line) in the left panel. Comparison with previous $\tau$ mass measurements and the PDG average value in the right panel.

\section{Prospects for Tau Lepton Flavor Violation}

According to the SM, lepton flavor is a conserved quantity. However, the discovery of neutrino oscillations established the non-conservation of lepton flavor in the neutral lepton sector. Hence, the question remains if the violation of lepton flavor also occur in the charged lepton sector. Charged LFV is so suppressed in the SM that, if observed, it would provide unambiguous evidence of physics beyond the SM.

Figure 4 shows prospects for the various LFV decay channels of the $\tau$ to be studied at Belle II. In particular, the golden channels for studying charged LFV are $\tau \rightarrow 3 \mu$ and $\tau \rightarrow \mu \gamma$. Belle II expects to push the current sensitivity further by more than one order of magnitude by using the full $50 \mathrm{ab}^{-1}$ data set.

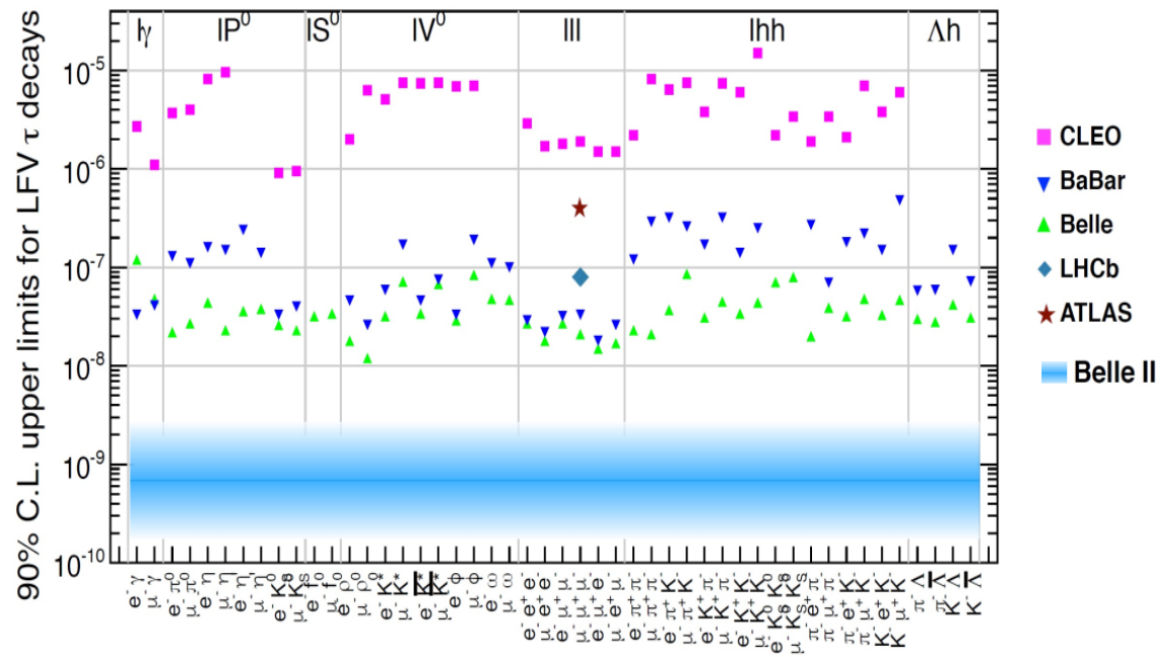

Figure 4: Current 90\% C.L. upper limits for the branching fraction of $\tau$ LFV decays. The blue bar indicates the Belle II prospects, assuming an integrated luminosity of $50 \mathrm{ab}^{-1}[6]$ and absence of signals. 


\subsection{Search for LFV Decays $\tau \rightarrow \ell \alpha$}

One relevant LFV decay of the $\tau$ lepton not listed in Figure 4 is $\tau \rightarrow \ell \alpha$, where $\ell$ is an electron or a muon, and $\alpha$ is a BSM particle that escapes undetected. This decay appears in several NP models including axionlike particles, or Z' gauge bosons [7-9], and it attracted renewed interest thanks to a recent proposal of a new approach [10].

The general idea is to search for a two body decay in the $\tau$ rest frame, where the momentum of the signal lepton will manifest as a peak with position depending of the mass of the $\alpha$ particle. In order to perform the Lorentz boost to this frame, the flight direction of the $\tau$ is needed. We follow the ARGUS method [11]. Here, the direction of the signal $\tau$ is approximated by the unit vector of the three-prong-system momentum in $3 \times 1$ prong events. Additionally, the $\tau$ energy is approximated by $E_{\tau}=\sqrt{s} / 2$, where $\sqrt{s}$ is the collision energy in the CM system. Hence, the boost to the $\tau$ pseudorest frame becomes possible.

A template based analysis is used to fit the lepton momentum spectrum in the $\tau$ pseudorest frame, and using the modified-frequentist CLs approach [12], the upper limit at 95\% C.L. on $\operatorname{Br}(\tau \rightarrow$ $e \alpha) / \operatorname{Br}(\tau \rightarrow e v \bar{v})$ is computed for seven different mass values of the $\alpha$ particle. Additionally, a different method, called Thrust method [13], was tested. For both methods, the upper limit results are similar. Results based on simulation are shown in Figure 5.

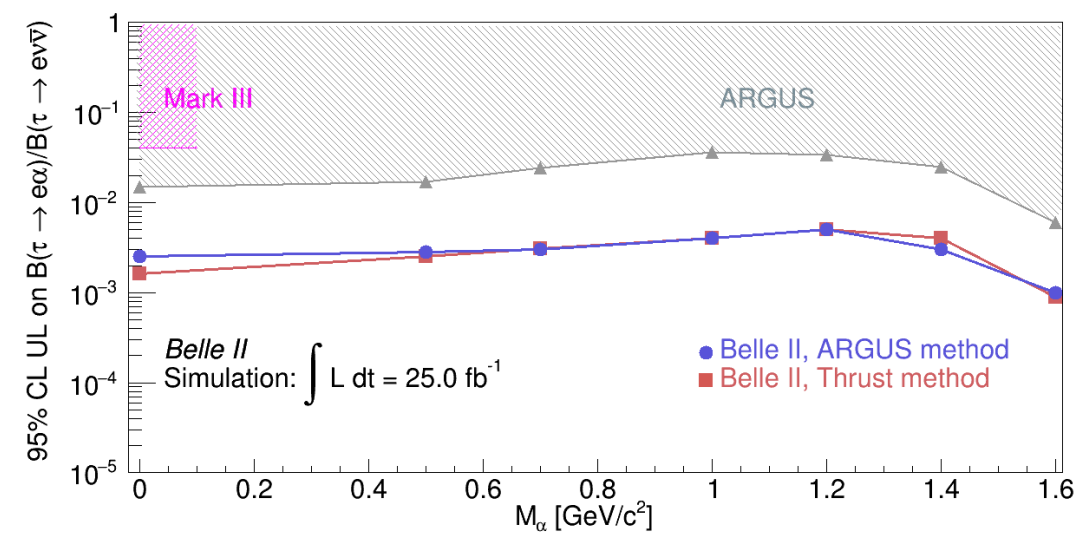

Figure 5: Upper limit estimation for $\operatorname{Br}(\tau \rightarrow e \alpha) / \operatorname{Br}(\tau \rightarrow e v \bar{v})$ using the ARGUS and Thrust methods, assuming an integrated luminosity of $25 \mathrm{fb}^{-1}$ of Belle II simulated data. Previous experimental results from ARGUS [11] and Mark II [14] collaborations are also shown. No systematic uncertainties are included.

\section{Conclusions}

The Belle II experiment has a rich program of precision measurements and searches for new physics in the $\tau$-lepton sector. Because of the low-background environment, the stringent production kinematic constraints, the high luminosity and the excellent detector capabilities, it is expected both to improve many of the current best results, and provide new ones in $\tau$ physics.

A number of analyses are already in advance state with the current luminosity. In the precision measurements, the preliminary result on the $\tau$ mass with early data shown that Belle II will provide the most precise $\tau$ mass measurement amongst the pseudomass techniques. In BSM physics 
searches, a first result on the lepton-flavor-violating decay channel $\tau \rightarrow \ell \alpha$ is expected in the near future.

Belle II expects to deliver unique results in high precision $\tau$ physics, becoming a major player in the near future and for years to come.

\section{References}

[1] Y. Ohnishi, T. Abe, T. Adachi, K. Akai, Y. Arimoto, K. Ebihara et al., Accelerator design at SuperKEKB, Progress of Theoretical and Experimental Physics 2013 (2013) .

[2] T. Abe, I. Adachi, K. Adamczyk, S. Ahn, H. Aihara, K. Akai et al., Belle II Technical Design Report, 1011.0352.

[3] A. Lusiani, Status and progress of the hflav-tau group activities, EPJ Web of Conferences 218 (2019) 05002.

[4] H. Albrecht, H. Ehrlichmann, T. Hamacher, R. Hofmann, T. Kirchhoff, A. Nau et al., A measurement of the tau mass, Physics Letters B 292 (1992) 221.

[5] Belle II Collaboration, $\tau$ lepton mass measurement at Belle II, 2008.04665.

[6] E. Kou, P. Urquijo, W. Altmannshofer, F. Beaujean, G. Bell, M. Beneke et al., The Belle II Physics Book, Progress of Theoretical and Experimental Physics 2019 (2019).

[7] B. Grinstein, J. Preskill and M.B. Wise, Neutrino masses and family symmetry, Physics Letters B 159 (1985) 57.

[8] J. Heeck, Lepton flavor violation with light vector bosons, Physics Letters B 758 (2016) 101.

[9] L. Calibbi, D. Redigolo, R. Ziegler and J. Zupan, Looking forward to lepton-flavor-violating ALPs, Journal of High Energy Physics 2021 (2021) .

[10] E. De La Cruz-Burelo, A. De Yta-Hernandez and M. Hernandez-Villanueva, New method for beyond the standard model invisible particle searches in tau lepton decays, Phys. Rev. D 102 (2020) 115001.

[11] ARGUS collaboration, A Search for lepton flavor violating decays $\tau \rightarrow e \alpha, \tau \rightarrow \mu \alpha, Z$. Phys. C68 (1995) 25.

[12] A.L. Read, Presentation of search results: the $C L_{s}$ technique, Journal of Physics G: Nuclear and Particle Physics 28 (2002) 2693.

[13] The Belle II Collaboration, Belle II experiment sensitivity to the LFV decay $\tau \rightarrow e+\alpha$, BELLE2-NOTE-PL-2020-018 (2020) .

[14] R.M. Baltrusaitis, J.J. Becker, G.T. Blaylock, J.S. Brown, K.O. Bunnell, T.H. Burnett et al., $\tau$ Leptonic Branching Ratios and a Search for Goldstone-Boson Decay, Phys. Rev. Lett. 55 (1985) 1842. 